\title{
Frenkel Line in Nitrogen Terminates at the Triple Point SUPPLEMENTARY INFORMATION
}

\author{
Ciprian G. Pruteanu*, Marcin Kirsz ${ }^{\dagger}$, and Graeme J. Ackland** \\ ${ }^{1}$ SUPA, School of Physics and Astronomy and Centre for Science at Extreme \\ Conditions, The University of Edinburgh, Edinburgh EH9 3FD, United Kingdom \\ *cip.pruteanu@ed.ac.uk \\ †marcin.kirsz@ed.ac.uk \\ **gjackland@ed.ac.uk
}

\section{ABSTRACT}

\section{SI to Frenkel Line in Nitrogen Terminates at the Triple Point}

Keywords: nitrogen, supercritical, molecular dynamics, liquids, high pressure

\section{CONTENTS}

This document contains:

- OPLS-AA Pair Distribution Functions

- OPLS-AA Coordination Numbers

- State of the OPLS-AA Forcefield at $160 \mathrm{~K}$

- Machine Learning Interatomic Potential for Nitrogen

- Pair Distribution Functions for ML Model

- Correlated Changes in Diffusion Constant and Coordination Number 


\section{OPLS-AA PAIR DISTRIBUTION FUNCTIONS}
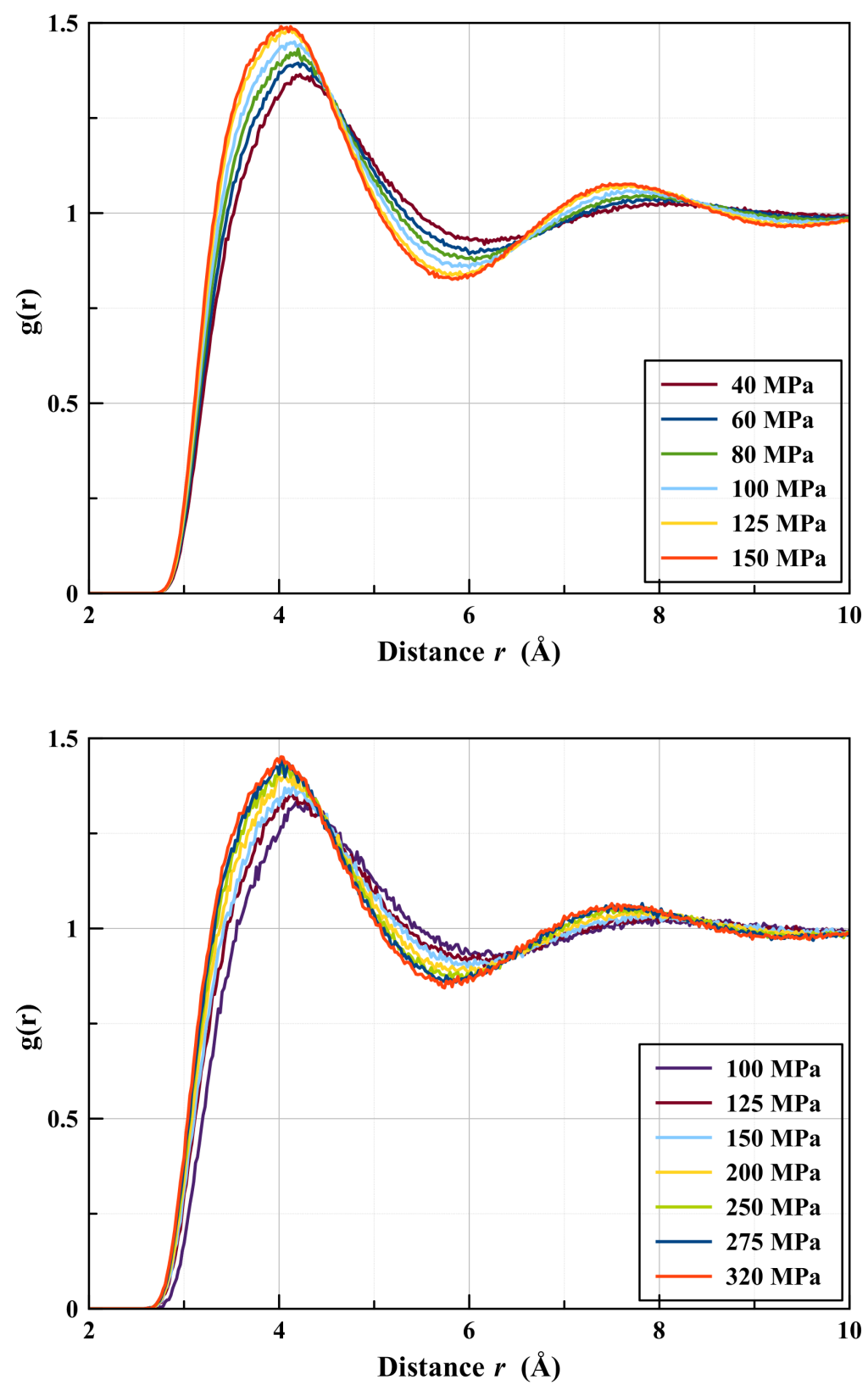

Figure 1. Pair Distribution functions for $\mathrm{N}_{2}$ obtained using the OPLS-AA model at $300 \mathrm{~K}$ (top) and $500 \mathrm{~K}$ (bottom). 


\section{OPLS-AA COORDINATION NUMBERS}

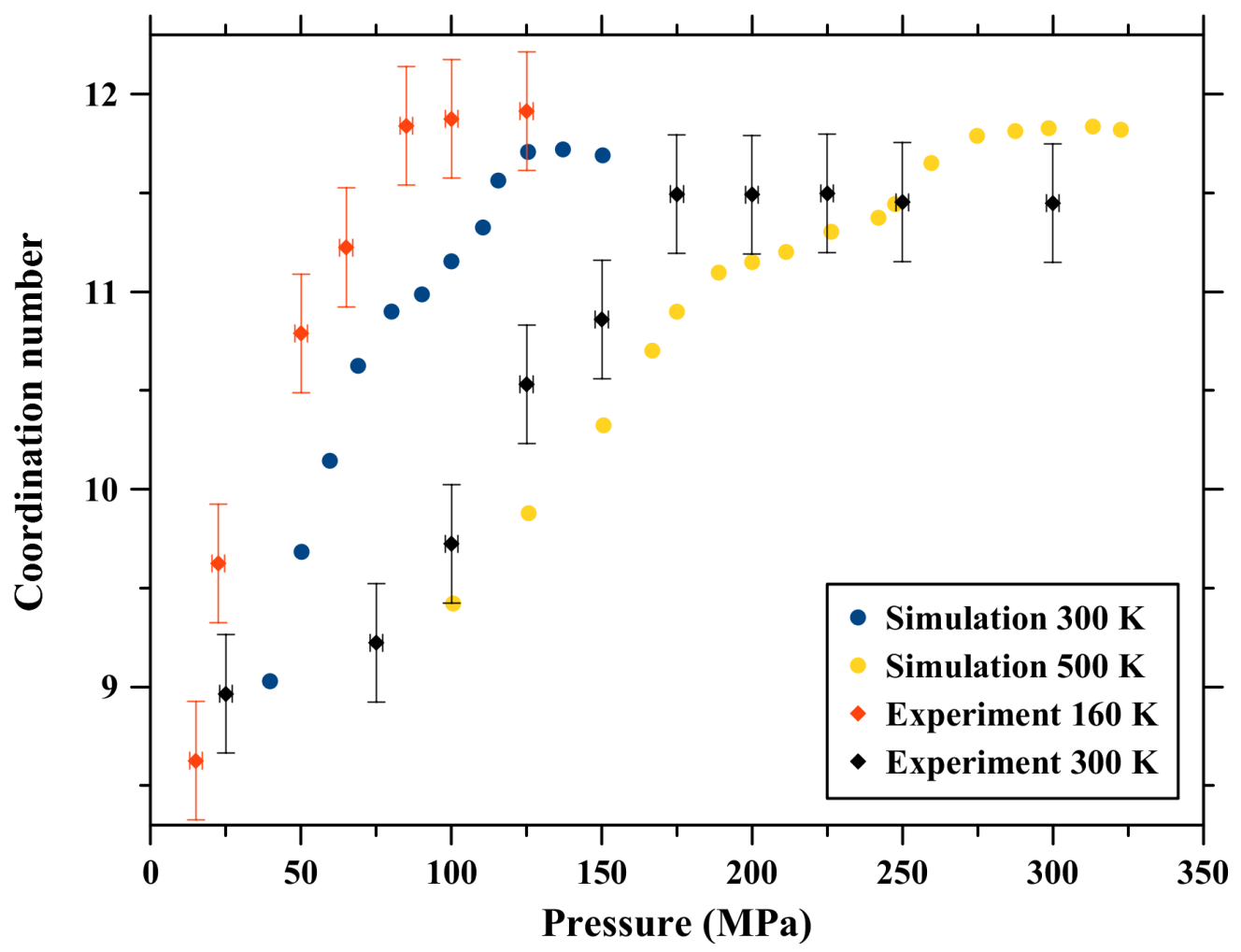

Figure 2. Coordination numbers for $\mathrm{N}_{2}$ obtained using the OPLS-AA model at $300 \mathrm{~K}$ and $500 \mathrm{~K}$. 


\section{STATE OF THE OPLS-AA FORCEFIELD AT $160 \mathrm{~K}$}

As there are no available studies on the phase diagram of our employed OPLS-AA $\mathrm{N}_{2}$ model, we had to first ensure the model was producing reasonable phases for comparison to the experimental data. We found that at $160 \mathrm{~K}$ unfortunately the model does not produce a stable liquid phase, NPT equlibration runs at various pressures yielding either a gas phase (pressures $\leq 2.65 \mathrm{MPa}$ ) or a solid non-crystalline phase (pressures $\geq 2.7$ $\mathrm{MPa}$ ). The justification of these statements is presented below, where vast jumps in the equilibrium density are readily visible when compressing from $2.65 \mathrm{MPa}$ to 2.7 $\mathrm{MPa}$. A visual inspection of the pair distribution functions also reveals a significantly more structured function above 2.7 MPa than those corresponding to fluid phases, and a significantly less structured function for pressures below 2.65 MPa.
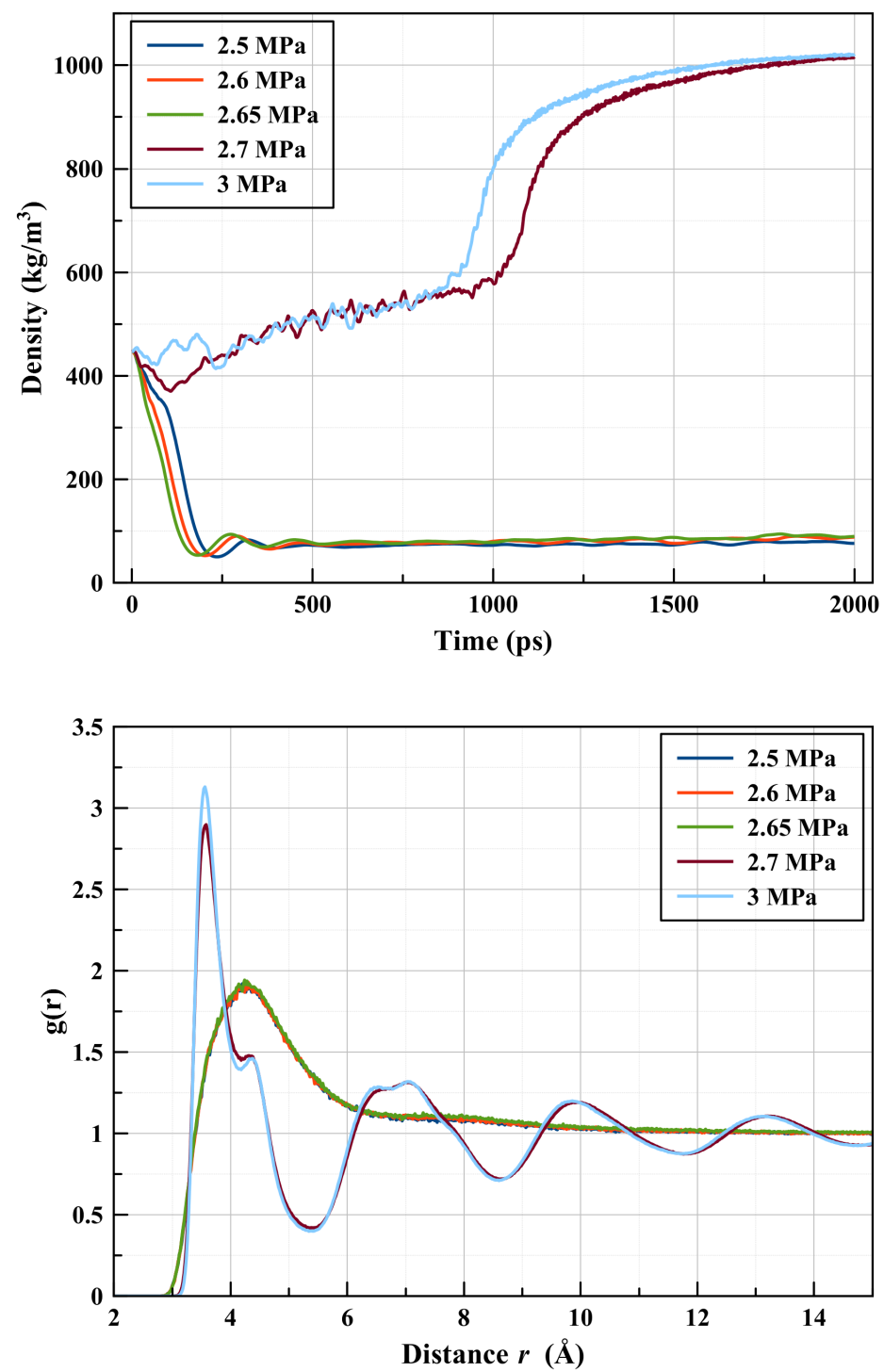

Figure 3. Evolution of the system density with time during NPT equilibration at $160 \mathrm{~K}$ (top) and pair distribution functions for the last $500 \mathrm{ps}$ of the runs (bottom). 
The mean square displacements and running coordination numbers presented below add further evidence on the absence of a liquid phase at $160 \mathrm{~K}$ in the employed OPLSAA forcefield. All quantities were sampled from the last 500 ps of the NPT run where in all cases the system density stabilized.
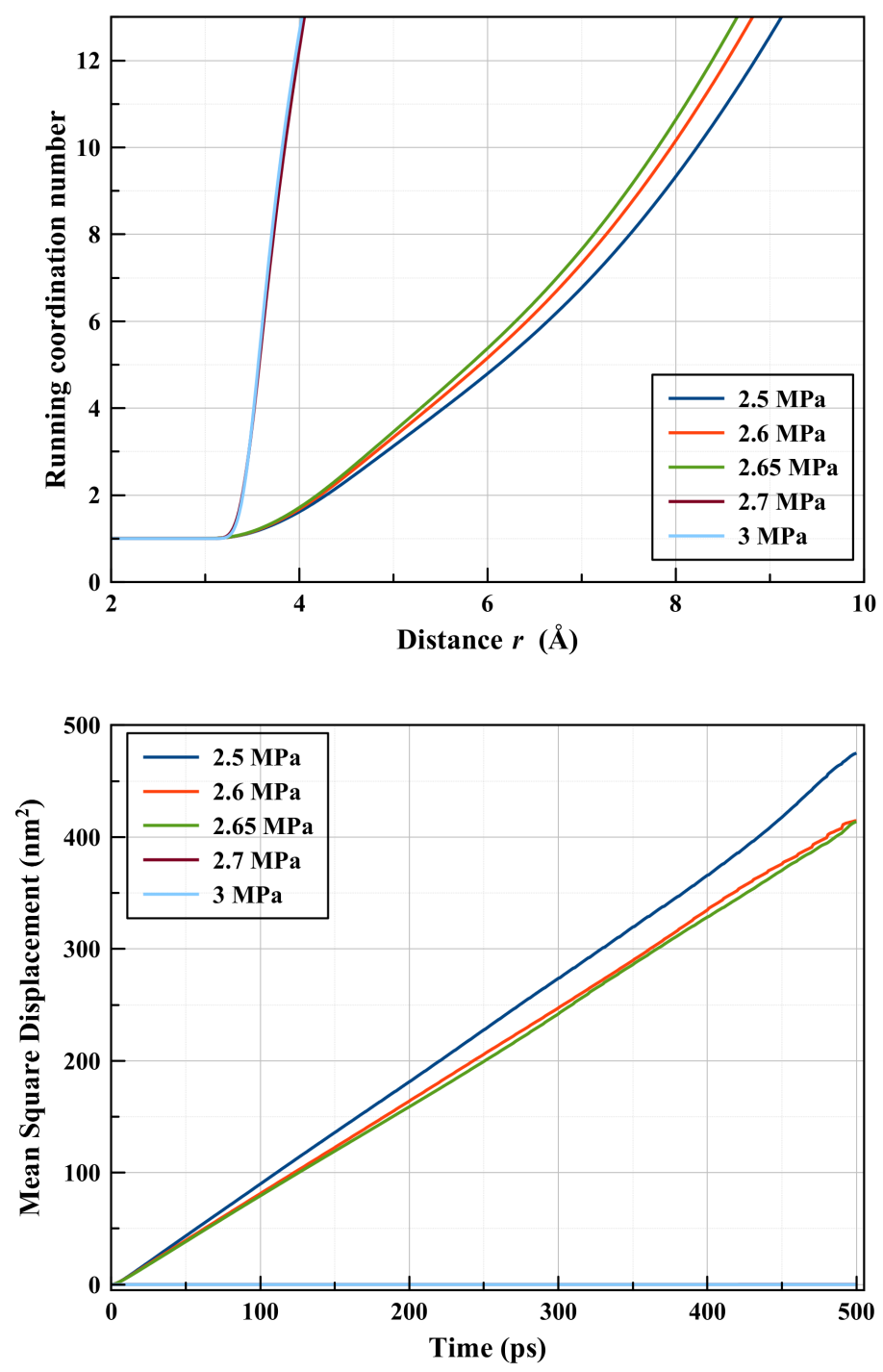

Figure 4. Running coordination numbers and mean square displacements for the OPLS-AA forcefield at $160 \mathrm{~K}$ for the last $500 \mathrm{ps}$ of NPT simulation. The abrupt jumps in coordination number between 2.65 and $2.7 \mathrm{MPa}$ shows the system does not move within a single fluid phase (where all changes would be continuous). 


\section{MACHINE LEARNING INTERATOMIC POTENTIAL FOR NI- TROGEN}

\subsection{Training Data}

The training data are generated using the ab initio pair potential based on calculations from the coupled-cluster method with single, double and noniterative triple excitations $\operatorname{CCSD}(\mathrm{T})$ [3]. The process of building the machine learning training database and potential is illustrated in fig. 5 and explained in the main text. We optimise our model to reproduce experimental equation of state and radial distribution function at a range of temperature-pressure conditions.

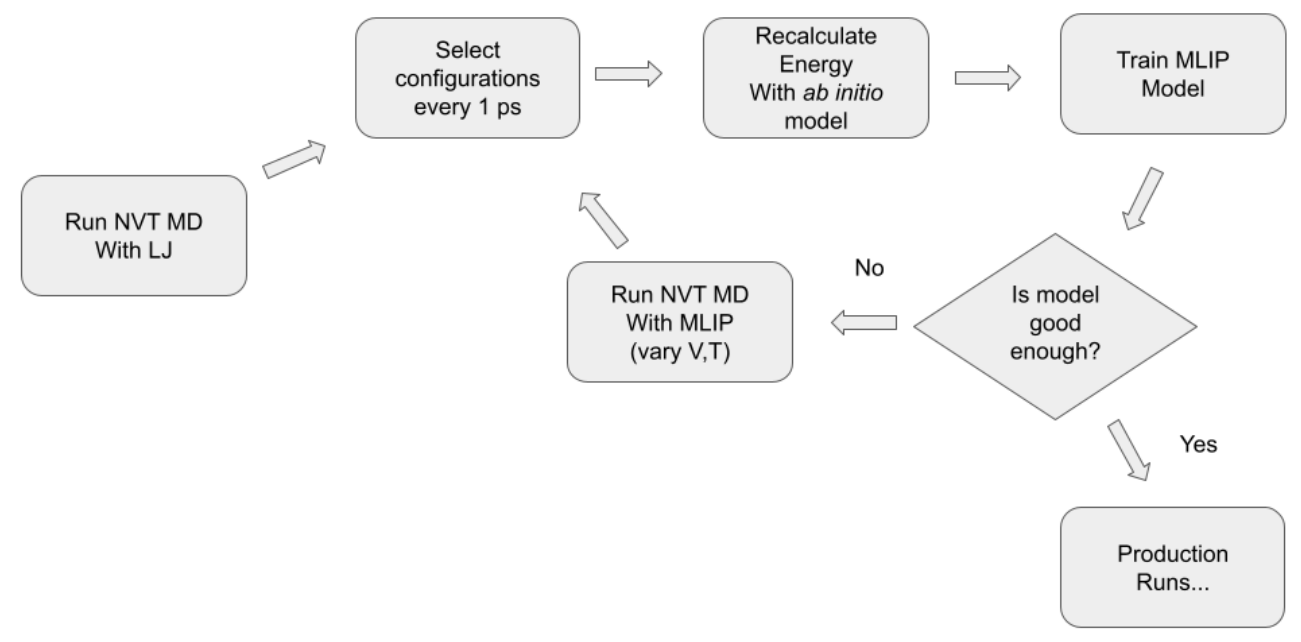

Figure 5. The iterative process of building machine learning interatomic potential for nitrogen.

\subsection{Descriptors}

The local atomic environment is described using a combination of two-body and manybody descriptors. For the pairwise descriptor we use localised blip basis functions given in [4] along with the commonly used cutoff function from [1]. The functional form of the two-body descriptor is given in eq. 1 where parameter $n$ controls the position of the blip function and the cutoff distance is set to $10 \AA$.

$$
v_{n}\left(r_{i j}\right)=B_{n}\left(r_{i j}\right) f_{c}\left(r_{i j}\right)
$$

The many-body descriptor is obtained by first computing density using Gaussian Type Orbitals (GTO) (eq. 2) similarly to [5] and [6]. Here $x, y, z$ are components of the displacement vector between two atoms, $\eta$ and $r_{s}$ control width and position of the Gaussian radial basis function respectively and $l_{x}, l_{y}, l_{z}$ are the quantised directionaldependent angular momenta.

$$
\psi_{l_{x}, l_{y}, l_{z}}^{\eta, r_{s}}\left(r_{i j}\right)=x^{l_{x}} y^{l_{y}} z^{l_{z}} \exp \left(-\eta\left|r_{i j}-r_{s}\right|^{2}\right)
$$


The many-body descriptor of the $\mathrm{i}$-th atom is then given by eq. 3 , where $L=$ $l_{x}+l_{y}+l_{z}$ is the quantised angular momentum which in our case is restricted to $L_{\max }=1$.

$$
\phi_{L, \eta, r_{s}}^{(i)}=\sum_{l_{x}, l_{y}, l_{z}}^{L} \frac{L !}{l_{x} ! l_{y} ! l_{z} !}\left(\sum_{j} \psi_{l_{x}, l_{y}, l_{z}}^{\eta, r_{s}}\left(r_{i j}\right)\right)^{2}
$$

The many-body descriptor captures up to three-body interactions (see [6] for details). To fully capture four dimensional potential energy surface of two interacting nitrogen molecules we introduce nonlinear transformation of the input descriptors during regression stage.

\subsection{Regression}

We use Bayesian approach to regularised linear regression [2], where the effective model complexity is controlled by the regularisation term. This approach avoids the usual problem of over-fitting when only maximum likelihood is used. The regularisation term is optimised automatically using training data alone with the expectation maximisation algorithm [2].

All descriptors are transformed with the polynomial basis functions $\chi_{j}(x)=x^{j}$ up to order of two. This "mixing" of descriptor components is essential to fully capture 4D PES of two nitrogen molecules.

\subsection{Model parameters and availability}

The model is available for download from https: / / ta-dah. readthedocs.io/ en/tadahvo/potentials.html. The potential file contains all model parameters. The model requires "Ta-dah!" - a machine learning library for the interatomic potentials development - to be installed and LAMMPS to be compiled with the provided interface. The library and LAMMPS interface are available from https: //ta-dah.readthedocs.io/en/tadahvo. 


\section{PAIR DISTRIBUTION FUNCTIONS FOR ML MODEL}
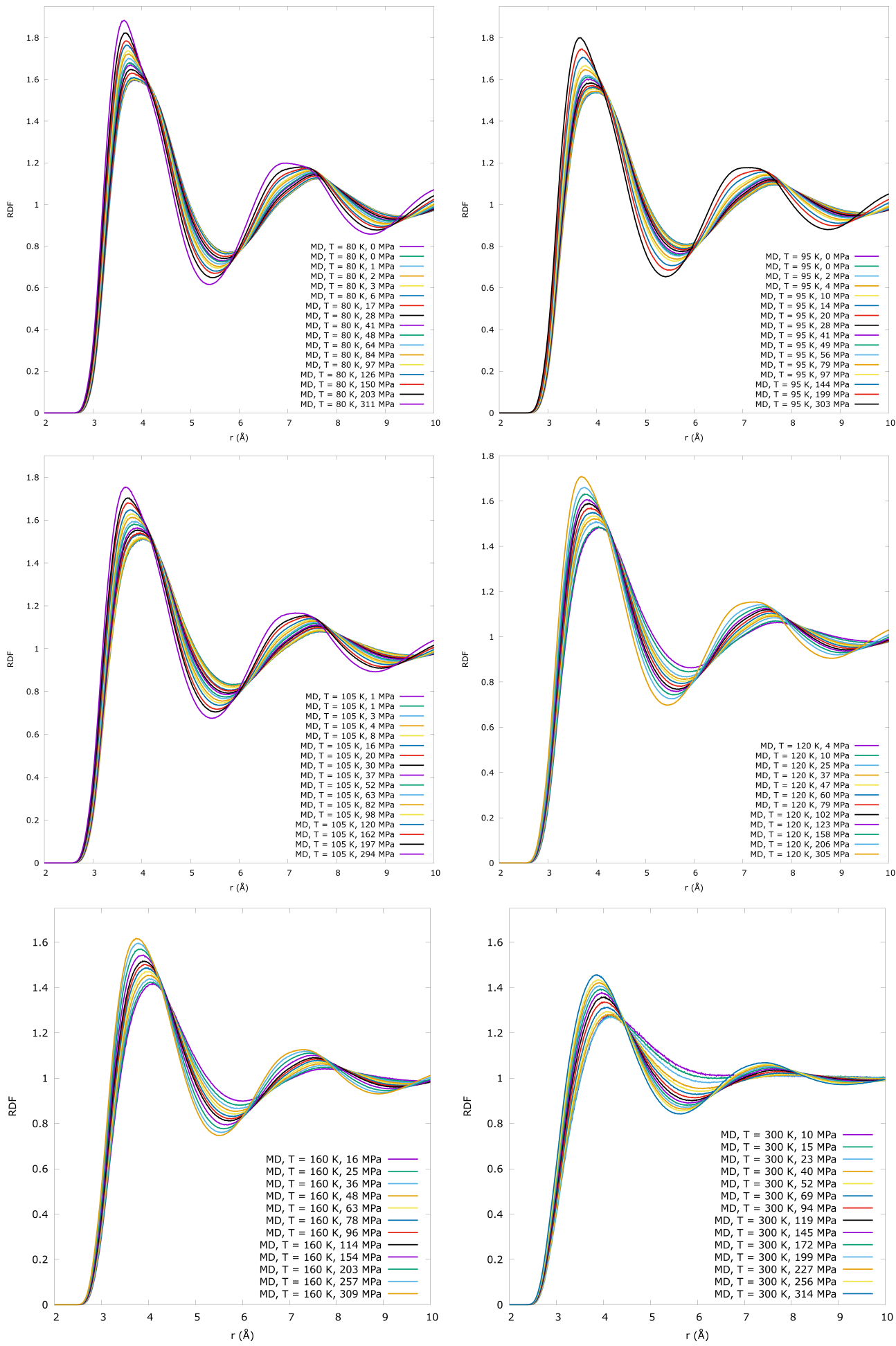

Figure 6. Radial Distribution Functions for $\mathrm{N}_{2} \mathrm{ML}$ model for all subcritical and supercritical isotherms followed in the present study. 


\section{CORRELATED CHANGES IN DIFFUSION CONSTANT AND COORDINATION NUMBER}
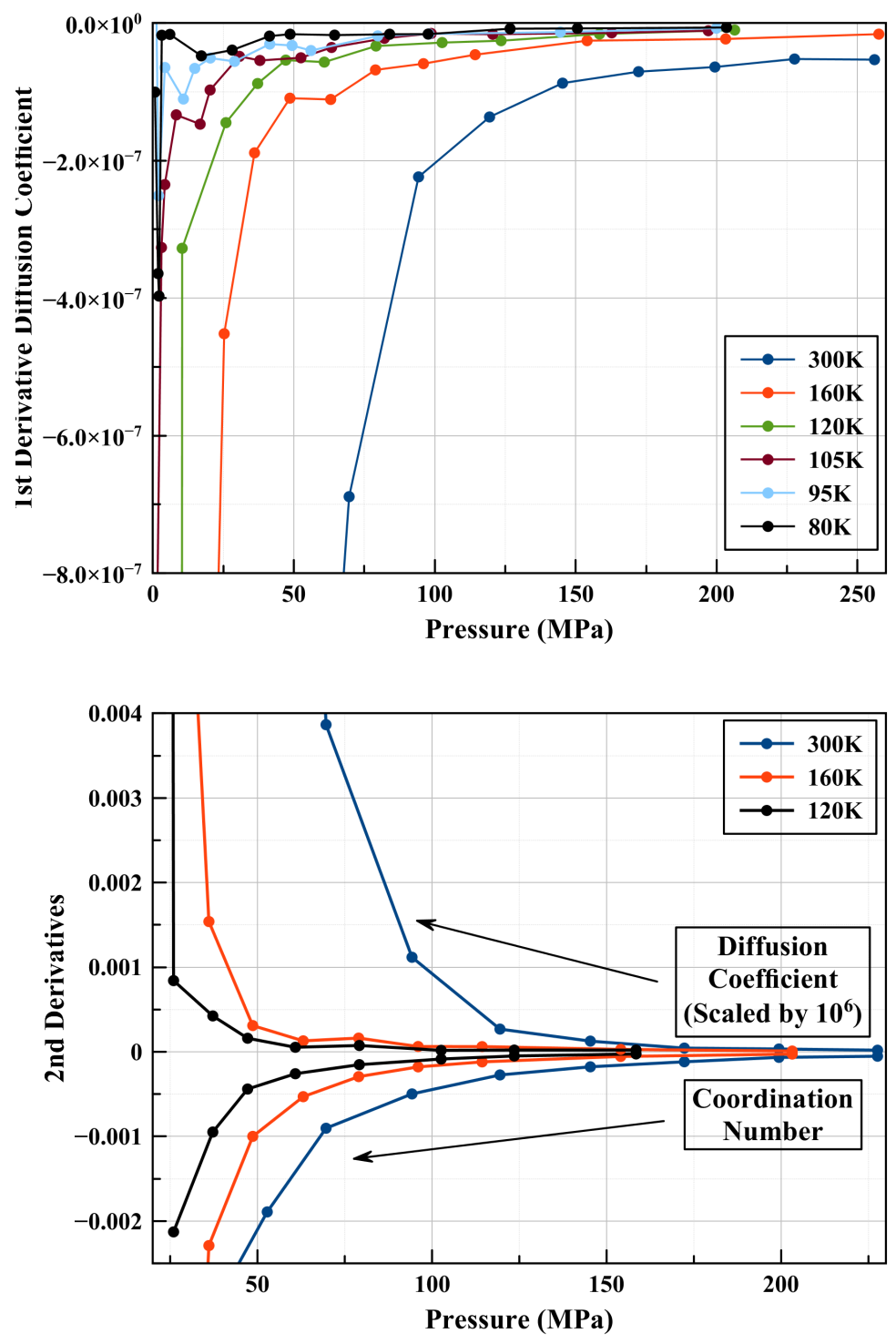

Figure 7. (Top) First derivative of the diffusion coefficient calculated as finite differences. (Bottom) Combined second derivatives of diffusion coefficient (scaled by $10^{6}$ ) and coordination numbers as functions of pressure, at 120, 160 and $300 \mathrm{~K}$. 


\section{REFERENCES}

[1] Behler, J. and Parrinello, M. (2007). Generalized Neural-network Representation of High-dimensional Potential-energy Surfaces. Physical Review Letters, 98(14):146401.

[2] Bishop, C. M. (2006). Machine Learning and Pattern Recoginiton. Springer-Verlag.

[3] Hellmann, R. (2013). Ab Initio Potential Energy Surface for the Nitrogen Molecule Pair and Thermophysical Properties of Nitrogen Gas. Molecular Physics, 111(3):387401.

[4] Hernández, E., Gillan, M., and Goringe, C. (1997). Basis Functions for Linearscaling First-principles Calculations. Physical Review B - Condensed Matter and Materials Physics, 55(20):13485-13493.

[5] Takahashi, A., Seko, A., and Tanaka, I. (2017). Conceptual and Practical Bases for the High Accuracy of Machine Learning Interatomic Potentials: Application to Elemental Titanium. Physical Review Materials, 1(6):063801.

[6] Zhang, Y., Hu, C., and Jiang, B. (2019). Embedded Atom Neural Network Potentials: Efficient and Accurate Machine Learning with a Physically Inspired Representation. Journal of Physical Chemistry Letters, 10(17):4962-4967. 